\title{
CARACTERÍSTICAS QUÍMICAS, FÍSICAS Y SENSORIALES DE UN QUESO DE CABRA ADAPTADO DEL TIPO "CROTTIN DE CHAVIGNOL"1
}

\author{
Alejandro Chacón-Villalobos ${ }^{2}$, María Lourdes Pineda-Castro ${ }^{3}$
}

\begin{abstract}
RESUMEN
Características químicas, físicas y sensoriales de un queso de cabra adaptado del tipo "Crottin de Chavignol". El objetivo del presente trabajo fue determinar la aceptación del público por un queso caprino previamente caracterizado adaptado del "Crottin de Chavignol" que fue producido en la zona de Tres Ríos, Cartago, Costa Rica, durante el segundo semestre del año 2006. Se elaboraron 10 lotes de queso con el procedimiento artesanal empleado en Tres Ríos de Cartago y con leche evaluada químicamente para proteína (p), lactosa (1), sólidos totales (st), sólidos no grasos (sng), sólidos grasos (g), peso específico(pe) y ácido láctico (al). Treinta muestras aleatorias fueron evaluadas fisicoquímicamente para contenido de humedad $(\mathrm{H})$, materia seca (MS), acidez (A), sal $(\mathrm{S})$, proteína $(\mathrm{P})$, grasa $(\mathrm{G})$ y color en las escalas CIELab y CIELch. Cincuenta muestras aleatorias se evaluaron con un análisis de perfil de textura abarcando dureza (D), elasticidad (E), cohesividad (C), masticabilidad (M) y adhesividad (AD). La calidad sensorial se comparó con la de un queso crema vacuno empleando una prueba sensorial afectiva seguida de un análisis de conglomerados de Ward. El queso obtenido presentó en promedio un rendimiento de 17,5\%. El $55 \%$ de los consumidores mostró igual aceptación por ambos quesos, mientras que a un $45 \%$ le desagrado el caprino.
\end{abstract}

\begin{abstract}
Chemical, physical and sensorial characteristics of goat cheese elaborated from a "Crottin de Chavignol" modified recipe. The objective of this work was to determine the acceptance by local consumers of a modified goat cheese characterized as "Crottin de Chavignol" and produced in Tres Rios, Cartago, Costa Rica during the second semester of 2006. Ten batches of a goat cheese were elaborated from a "Crottin de Chavignol" modified recipe. Milk quality parameters were: total protein (p), lactose (l), solids (s), non fat solids (nfs), fat (f), specific weight (sw) and lactic acid (la). Thirty random samples were evaluated according to moisture content (M), dry matter (DM), acidity (A), salt $(\mathrm{S})$, protein $(\mathrm{P})$, fat $(\mathrm{F})$ and color at CIELab and CIELch scales. Fifty random samples were evaluated with a texture profile analysis (TPA) including hardness $(\mathrm{H})$, elasticity $(\mathrm{E})$, cohesivity $(\mathrm{C})$, chewiness $(\mathrm{CH})$ and adhesiveness (AD). Consumer sensorial evaluation of goat cheese was compared with a bovine cheese cream using an affective sensorial test followed by a Ward clusters test. Cheese yield was $17.5 \%$. About $55 \%$ of the consumer accepted both types of cheese, while a $45 \%$ indicated displeasure toward the goat cheese.
\end{abstract}

Key words: Goat milk, dairy, composition, texture, acceptance.

Palabras claves: Leche de cabra, lácteos, composición, textura, aceptación.

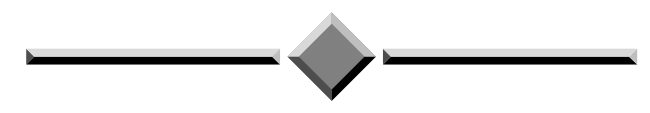

\footnotetext{
1 Recibido: 14 de enero, 2009. Aceptado: 16 de noviembre, 2009. Proyecto inscrito en la Vicerrectoría de Investigación No.737-A4-040, Universidad de Costa Rica.

2 Estación Experimental Alfredo Volio Mata, Universidad de Costa Rica. Cartago, Costa Rica. alejandro.chacon@ucr.ac.cr

3 Escuela de Tecnología de Alimentos, Universidad de Costa Rica. San José, Costa Rica. maria.pinedacastro@ucr.ac.cr
} 


\section{INTRODUCCIÓN}

La industrialización de los productos de la leche constituye uno de los sectores agroindustriales más representativos y en constante crecimiento de la economía costarricense (Vega 2002, Díaz 2004), y uno de los procesos más dinámicos de América Latina (Morón et al. 2005). No obstante, el sector lácteo del país se fundamenta casi en forma exclusiva en derivados de la leche de vaca (Corrales y Chacón 2005, Rojas et al. 2007, Vargas et al. 2007); los derivados de leche de otras especies menos tradicionales, como la de cabra, están supeditados al sector artesanal, con limitadas cadenas de distribución y venta (Chacón 2004). Los pocos productos existentes en el mercado, destacándose principalmente la leche fluida, tienen prejuicios sensoriales y culturales y sufren de un desconocimiento derivado de su poca disponibilidad, aspectos que provocan que sean pocos los consumidores dispuestos a pagar precios elevados para adquirir estos productos, usualmente en el mismo lugar donde se manufacturan (Chacón et al. 2008).

El aislamiento derivado de una comercialización limitada, ya sea por falta de mercados o por cadenas de distribución deficientes, es una de las causas que originan la aparición de derivados lácteos artesanales de consumo muy regional (Brito 1985). Factores como el anterior explican por qué países como Francia, Grecia, España, Italia y Suecia se han caracterizado por producir distintos quesos a partir de leche de cabra (Kozhev et al. 1998). Entre éstos puede citarse el queso con denominación de origen "Crottin de Chavignol", producido desde el siglo XVI al sur de Francia empleando cuajada cruda de leche entera de cabra; éste se caracteriza por ser un queso semi madurado o incluso madurado de pasta blanda y desuerado espontáneo (Tacsan 1987). Este queso, con contenido de grasa de al menos $45 \%$, tiene la forma de un cilindro plano de alrededor de $140 \mathrm{~g}$ de peso y con un color muy blanco en su estado fresco, disminuyendo el peso, acentuándose el sabor "caprino" característico y tornándose amarillento hacia el final de su fase de maduración que se da alrededor de los cuatro meses (Devoyod 1991). Posee una pasta semiblanda, seca y consistente en su estado fresco, tornándose la misma semidura cuando está avanzada la maduración (Tacsan 1987).

Trabajos para diversificar el sector caprinocultor costarricense emprendidos por Tacsan (1987), introdujeron en la zona de Ochomogo de Cartago un queso derivado del "Crottin de Chavignol" reformulado y más adaptado al gusto del consumidor nacional. En poco tiempo esta formulación original fue sufriendo algunas variaciones, dada la artesanalidad del manejo y de la oralidad en la divulgación del proceso entre los productores, hasta convertirse 20 años después en un producto muy peculiar que guarda similitudes muy generales con su antecesor francés. Esfuerzos efectuados en la Estación Experimental de Ganado Lechero Alfredo Volio Mata de la Universidad de Costa Rica en el año 2002, permitieron tipificar el proceso actual de manufactura efectuado por los artesanos. El queso cilíndrico que se obtiene de dicho proceso se comercializa usualmente fresco y, al igual que su antecesor, es de pasta blanda blanca obtenida posteriormente a un desuerado espontáneo. Se diferencia del producto original, entre otros aspectos, en que no hay una maduración, en un menor tiempo de acidificación de la cuajada, así como que se prescinde del uso de Penicillium candidum.

El estudio de quesos regionales incipientes es importante desde un punto de vista comercial (Díaz 2004), especialmente en segmentos de mercado deprimidos como el caprinocultor (Brito 1989), lo cual hace relevante investigaciones como la presente. En este tipo de caracterizaciones, aspectos químicos como el contenido de grasa, proteína, sal y la acidez son de interés primordial al momento de evaluar la calidad y la reología de un queso (Scholz 1995).

La acidez en un queso no solo tiene incidencia sobre el sabor, sino que además influencia directamente los cambios que experimenta la red de proteína que constituye la cuajada del queso, teniendo ésto un papel en los fenómenos de sinéresis y textura final (Pinho et al. 2004). Un pH cercano al punto isoeléctrico provoca fuerzas iónicas e hidrófobas fuertes que resultan en una red de caseína compacta típica de los quesos duros, mientras que en el caso de un pH más alto las caseínas presentan una carga negativa, lo que genera repulsión entre los agregados proteicos, generándose un queso con mayor absorción de agua, más elasticidad y menos compacto (Adda et al. 1982). La sinéresis no es únicamente afectada por la acidez de un queso, también es determinada por las circunstancias mismas del proceso de elaboración y por la presencia de calcio libre que provoca la unión de la caseína en la red proteica de la cuajada (Walstra et al. 1999). La humedad remanente en un queso es un factor determinante en la textura final, donde bajos contenidos se asocian con quesos duros y poco elásticos (Adda et al. 1982). 
Además de tener un papel en el sabor y en la preservación de un queso, la sal en altas concentraciones disminuye la actividad enzimática proteolítica a la vez que incrementan la presión osmótica, lo que elimina parte del agua atrapada en la red proteica de la cuajada (Pinho et al. 2004). La grasa en los quesos generalmente actúa como material de relleno en la matriz de proteína, otorgando, cuando está presente en alto porcentaje, mayor elasticidad y menor firmeza, mientras que cuando su porcentaje disminuye se obtienen quesos más duros y rígidos (Küçüköner y Haque 2006, Theophilou y Wilbey 2007). Además de las interacciones antes descritas con la acidez, la proteína también afecta la dureza de un queso del mismo modo que la grasa, siendo los altos contenidos proteicos sinónimo de dureza (Walstra et al. 1999).

La composición de un queso es un importante determinante de las características de textura del mismo, aspecto que junto con el color y el sabor son variables importantes de consideración inmediata por parte de los consumidores como criterios de decisión de compra utilizados al establecer la calidad de un queso (Pinho et al. 2004). No obstante, aspectos como la tecnología aplicada, los cultivos iniciadores y las condiciones de maduración tienen mayor impacto en la textura del queso que su composición original (Walstra et al. 1999).

Los quesos caprinos presentan en términos generales sabores muy característicos, generados principalmente por ácidos grasos como el caprílico, caproico y cáprico, los cuales pueden llegar a resultar demasiado intensos para consumidores no habituados a este tipo de productos (Brito 1989). Es por esta razón que la evaluación de la aceptación sensorial de los quesos es tan importante en términos de mercadeo y segmentación de mercados (Corrales y Chacón 2005). Dichas evaluaciones se efectúan por medio del uso de escalas hedónicas con base en paneles de consumidores no entrenados (Carpenter et al. 2000).

La textura de un queso es un atributo sensorial que resulta de la combinación de propiedades físicas que son percibidas por los sentidos de la vista, el tacto y hasta el oído (Pinho et al. 2004). A pesar de que esta experiencia sensorial no puede ser completamente duplicada por ningún procedimiento instrumental, estos últimos son considerablemente menos costosos y consumen menos tiempo que las pruebas sensoriales, siendo fidedignamente correlacionables con atributos sensoriales críticos que permiten estimar la aceptabilidad por parte del consumidor (Steffe 1996). Las propiedades mecánicas se manifiestan por la reacción del queso al estrés provocado por una presión ejercida desde un texturómetro, lo cual simula la fuerza de masticación (Pinho et al. 2004). Este aparato permite establecer la fuerza necesaria para efectuar una prueba de compresión en dos ciclos en función del tiempo, lo cual se denomina análisis del perfil de textura (TPA, por sus siglas en inglés) (Bourne 2002).

La evaluación del color es un criterio muy variable que depende de numerosos factores, por lo cual es posible el uso de sistemas instrumentales que permitan obtener mediciones objetivas y estandarizadas (Pinho et al. 2004). Para este efecto se emplea un colorímetro que mide la luz reflejada por el alimento por medio de un foto detector, codificando esta señal en términos de algún sistema de medición lumínico de espacio polar como es el caso de la Escala de CIE (HunterLab 2008).

El objetivo del presente trabajo fue determinar la aceptación del público a un queso caprino previamente caracterizado adaptado del "Crottin de Chavignol" que es producido en la zona de Tres Ríos, Cartago, Costa Rica.

\section{MATERIALES Y MÉTODOS}

\section{Localización}

Este estudio se realizó durante el segundo semestre del año 2006. La preparación de los quesos, los análisis fisicoquímicos y las evaluaciones sensoriales se efectuaron en el laboratorio de química y módulo lácteo de la Estación Experimental de Ganado Lechero Alfredo Volio Mata de la Universidad de Costa Rica (EEAVM), ubicada en el Alto de Ochomogo de Cartago. En este lugar, ubicado a $1.542 \mathrm{msnm}$, la temperatura y la humedad relativa anual media son de 19,5 ${ }^{\circ} \mathrm{C}$ y del $84 \%$, respectivamente. Las evaluaciones de textura y color se efectuaron en el laboratorio de química de la Escuela de Tecnología de Alimentos de la Universidad de Costa Rica, ubicada en la sede Rodrigo Facio, en San Pedro de Montes de Oca, San José.

\section{Caracterización de la leche caprina y elaboración del queso}

Para la elaboración del queso tipo "Crottin" se siguió la formulación correspondiente al proceso 
artesanal efectuado por los pequeños productores de la zona de Cartago, Costa Rica, el cual fue caracterizado y estandarizado preliminarmente por la EEAVM (Chacón $2007)^{4}$, y ejecutado bajo condiciones de inocuidad garantizadas, extremando las medidas higiénicas y empleando una dotación técnica construida en acero inoxidable. Las cantidades elaboradas y el número de repeticiones se discuten en las secciones de análisis fisicoquímico y de diseño experimental.

Se utilizó en todas las elaboraciones leche fresca e íntegra de cabra (Capra hircus) de la raza "Lamancha", procedente del hato caprino de la EEAVM y con menos de tres horas de ordeño, el cual se efectuó en todos los casos a las 6:00 am. Los diferentes lotes de leche fueron analizados por el método Snap (González $2003)^{5}$ para garantizar la inexistencia de antibióticos. Además se evaluó la Acidez Titulable Expresada como Ácido Láctico (ATECAL) y el peso específico por medio de las metodologías citadas por Chacón (2004) y Kirk et al. (1999). Lo anterior para garantizar en todos los casos una materia prima con un valor de ATECAL menor a $0,18 \%$ y un peso específico igual o superior a 1,028 , criterios de calidad recomendados por la bibliografía para la leche destinada a la elaboración de derivados lácteos (Chacón 2003). Se evaluó adicionalmente en forma instrumental el contenido de lactosa, grasa y proteína de la leche utilizada mediante espectroscopia infrarroja empleando un analizador EKO-MILK ${ }^{\circledast}$ modelo standard. A partir de los datos de peso específico y porcentaje de grasa se estableció el contenido de sólidos totales y no grasos, utilizando la fórmula modificada de Richmond para leche fresca, tal y como establecen Kirk et al. (1999).

La leche, cuya calidad se garantizó por medio de las determinaciones antes descritas, se mantuvo en refrigeración a una temperatura de $4{ }^{\circ} \mathrm{C}$ hasta iniciar su procesamiento. En ese momento, la misma fue filtrada e inmediatamente pasteurizada por medio de un proceso LTLT $\left(65^{\circ} \mathrm{C} / 30\right.$ minutos) empleando una pasteurizadora eléctrica tipo "marmita". Posteriormente, la leche fue inoculada en las cantidades recomendadas por el fabricante con un cultivo láctico liofilizado FLDAN de la casa comercial CHr-Hansen, el cual contiene Lactococcus lactis subsp. cremoris, Lactoccous

4 Chacón, A. 2007. Proceso artesanal del queso tipo Crottin. EEAVM. Cartago, Costa Rica. Comunicación personal.

5 González, A. 2003. Evaluación de antibióticos en leche empleando el método Snap. INDULAC. San José, Costa Rica. Comunicación personal. lactis subsp. lactis, Leuconostoc mesenteroides subsp. Cremoris y Lactococcus lactis subsp. Diacetilactis. Se permitió un periodo de maduración de 30 minutos a 38 ${ }^{\circ} \mathrm{C}$. Seguidamente, a la leche se le adicionó $\mathrm{CaCl}_{2} \mathrm{Cal}-$ Sol CHr-Hansen en solución al $50 \%(0,5 \mathrm{ml} / 1$ leche $)$ y a continuación se coaguló a $37{ }^{\circ} \mathrm{C}$ durante 45 minutos empleando renina comercial CHY-MAX CHr-Hansen (1 ml/15 1 leche).

Finalizada la coagulación, la cuajada formada no se corta, permitiéndole a la misma permanecer íntegra y en reposo en el interior de la tina de coagulación por un período de 20 horas a temperatura ambiente. Al transcurrir este lapso de tiempo, la cuajada acidificada es transferida directamente sin cortar al interior de moldes plásticos cilíndricos de $14 \mathrm{~cm}$ de altura por 7 $\mathrm{cm}$ de diámetro con perforaciones de $3 \mathrm{~mm}$ de diámetro, separadas entre sí a $2,54 \mathrm{~cm}$ horizontalmente y 1,5 $\mathrm{cm}$ verticalmente. Seguidamente se permitió un desuerado por gravedad durante 20 horas a temperatura ambiente (Figura 1).
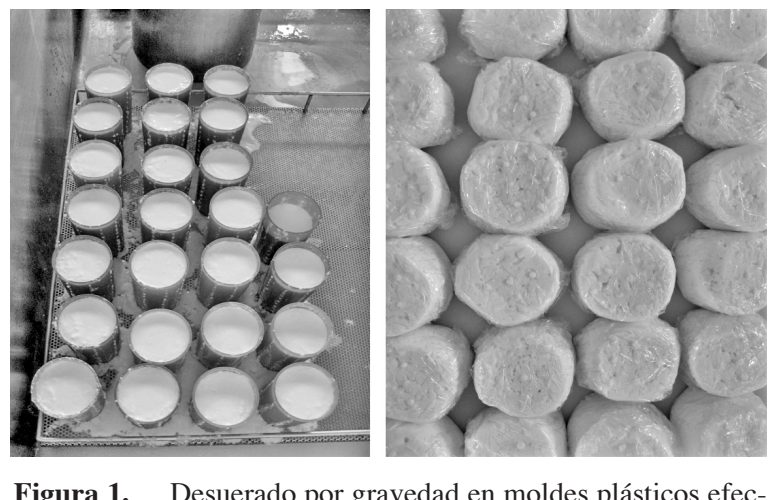

Figura 1. Desuerado por gravedad en moldes plásticos efectuado durante la manufactura del queso de cabra (izquierda), y producto obtenido al final (derecha). San José, Costa Rica. 2008.

Posteriormente, la pasta formada se voltea en el interior del molde y cada cara se sala superficialmente a discreción, empleando sal desecada. Se obtiene por lo general en este punto un queso cilíndrico pequeño de $5 \mathrm{~cm}$ de diámetro y $4 \mathrm{~cm}$ de altura, muy similar en dimensiones al "Crottin" original (Tacsan 1987). La pasta salada obtenida en cada molde se pesó y se empacó a continuación con una película plástica de polietileno de baja densidad, almacenándose durante tres días a $7{ }^{\circ} \mathrm{C}$ en una cámara refrigerada. Los 
rendimientos, con base en la leche fresca empleada y la masa de queso obtenida, se calcularon en todos los lotes experimentales posteriormente al salado. El proceso simplificado se resume en la Figura 2.

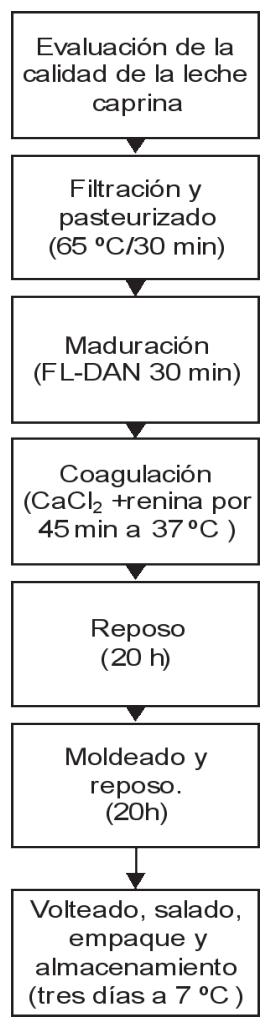

Figura 2. Flujo de proceso para la elaboración del queso tipo "Crottin". San José, Costa Rica. 2008.

\section{Evaluación química}

Se evaluaron por triplicado aleatorio los quesos generados en 10 lotes distintos para un total de 30 muestras (tres por lote), que fueron elaborados según metodología previamente descrita, constituyendo cada lote una repetición del proceso.

Los contenidos de humedad y la materia seca de todas las muestras de queso evaluadas se determinaron expresando la pérdida de peso por evaporación de agua como humedad mediante el método 9.26.08 de la AOAC (2000). La acidez fue expresada como porcentaje de ácido láctico titulado con $\mathrm{NaOH} 0,1 \mathrm{~N}$, empleando el método volumétrico 16.267 de la AOAC (2000). El contenido de sal se determinó por medio del método volumétrico de titulación para quesos 975,20 de la AOAC (2000). El contenido de proteína se obtuvo por medio de la determinación del total de nitrógeno, según el método 920.23 de la AOAC (2000). La grasa total se determinó por hidrólisis ácida, según describen Kirk et al. (1999).

\section{Evaluación del color}

La determinación de color de los quesos se realizó con un colorímetro HunterLab modelo ColorFlex ${ }^{\circledR}$ (Figura 3), el iluminante D65 y un ángulo de observación de $10^{\circ}$ (HunterLab, 2008). El colorímetro se calibró previamente a su uso empleando una teja negra y una teja blanca, y la correcta calibración del equipo se corroboró con una teja verde, según se describe en el manual de operación. Para cada muestra se obtuvieron las variables $L^{*}, a^{*}, b^{*}, C^{*}$ y $h^{*}$ del sistema de color de espacio polar CIE. El parámetro $\mathrm{L}^{*}$ indica variación en la luminosidad (variando desde $0 \%$ para negro y $100 \%$ para blanco), a* indica del mismo modo variación entre el verde (-a) y rojo (+a), b* señala variación entre el azul (-b) y el amarillo (+b), C* corresponde a la saturación del color, mientras que $\mathrm{h}^{*}$ hace referencia al ángulo de tonalidad. Se evaluaron por triplicado aleatorio los quesos generados en los 10 lotes experimentales para un total de 30 muestras.
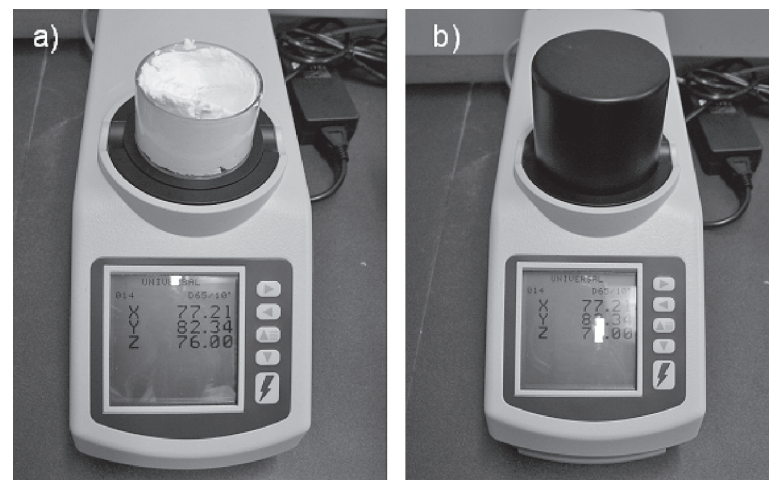

Figura 3. Análisis de color del queso tipo Crottin en el colorímetro Hunter Lab ColorFlex ${ }^{\circledR}$. a) muestra de queso colocada en la cubeta de medición, b) muestra cubierta con un fondo negro durante la determinación. San José, Costa Rica. 2008. 


\section{Evaluación de la textura}

La textura de las muestras se determinó con el texturómetro TA.XT Plus de Stable Micro Systems, empleando el software Exponent versión 4.0.8.0. Se aplicó la secuencia de análisis de perfil de textura (compresión en dos ciclos) utilizando una celda de carga de $50 \mathrm{~kg}$. Se evaluaron los 10 lotes experimentales de queso por quintuplicado, para un total de 50 muestras, tal y como recomiendan Pinho et al. (2004); éstas se mantuvieron en refrigeración a $7{ }^{\circ} \mathrm{C}$ hasta el momento del análisis.

Cada queso, con un diámetro aproximado de 7 $\mathrm{cm}$, se penetró un $\mathrm{cm}$ utilizando una celda cilíndrica de $3,5 \mathrm{~cm}$ (Figura 4). La velocidad de pre prueba fue de $120 \mathrm{~mm} / \mathrm{min}$ y las velocidades de prueba y post prueba fueron de $60 \mathrm{~mm} / \mathrm{min}$; el tiempo entre ciclos fue de $5 \mathrm{~s}$ y la fuerza de gatillo fue de $5^{*} 10^{-2} \mathrm{~N}$. Previo a la prueba, a cada queso se le removió con un cuchillo una capa de 3 a $5 \mathrm{~mm}$ con el fin de que la celda cilíndrica hiciera contacto con una superficie plana y nivelada. Se utilizó una celda más pequeña que la muestra (prueba de penetración) para realizar el TPA debido a que los quesos, por ser producidos de manera artesanal, varían mucho en su diámetro, por lo que se hubiera generado una alta variabilidad en los resultados si cada queso se compresionaba completamente; por otro lado, de la muestra no se pueden extraer trozos más pequeños pues, al cortarlo, la estructura del queso se debilita y tiende a desboronarse.
A partir del análisis de perfil de textura se calcularon los parámetros de dureza, cohesividad, adhesividad, elasticidad y masticabilidad (Armero y Collar 1997, Bara-Herczegh et al. 2002).

\section{Prueba sensorial afectiva}

La aceptación general del queso de cabra tipo "Crottin" se comparó con la de un queso crema de venta comercial común en Costa Rica, y elaborado a partir de leche de vaca, mediante una prueba sensorial afectiva, empleando un panel no entrenado de 52 consumidores y una escala hedónica semiestructurada de $100 \mathrm{~mm}$ (Villanueva et al. 2005). El propósito de esta determinación fue evaluar el desempeño sensorial comparativo del queso caprino con un producto vacuno similar más establecido y disponible en el mercado.

\section{Análisis estadístico}

Para cada uno de los parámetros químicos y físicos evaluados se calculó el promedio general de los datos con su respectiva desviación estándar.

Los datos de la prueba sensorial afectiva se sometieron a un análisis de conglomerados por el método de Ward, con el que se obtuvieron cuatro conglomerados de consumidores, de los cuales se eliminaron dos por contener menos de seis jueces. Al final se utilizaron los datos de 44 consumidores habituales. A los datos se les aplicó un análisis de varianza anidado por conglomerado.
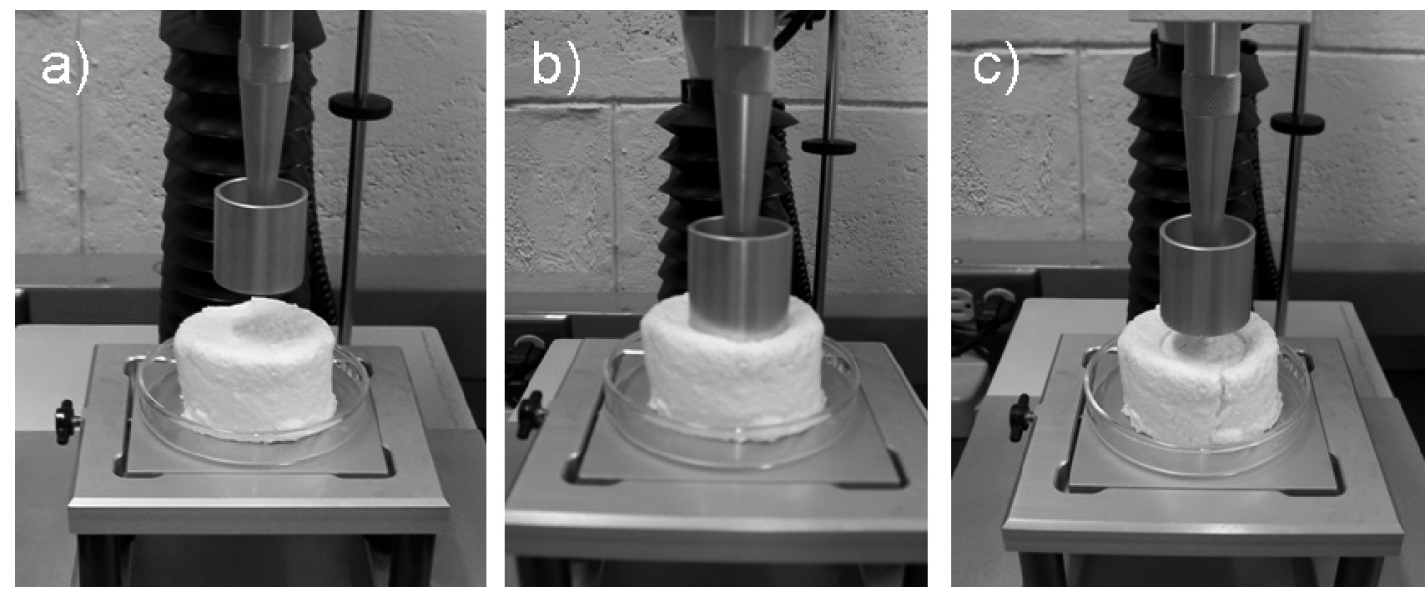

Figura 4. Análisis de la textura del queso durante el primer ciclo de compresión. a) pre prueba, b) prueba, c) post prueba. San José, Costa Rica. 2008. 


\section{RESULTADOS Y DISCUSIÓN}

\section{Evaluación química de la leche de cabra}

El Cuadro 1 presenta las características composicionales promedio de la leche de cabra empleada en la elaboración del queso en estudio.

Cuadro 1. Composición promedio de la leche de cabra empleada en la elaboración del queso de cabra. San José, Costa Rica. 2008.

\begin{tabular}{lcc}
\hline $\begin{array}{c}\text { Parámetro } \\
\text { evaluado }\end{array}$ & $\begin{array}{c}\text { Composición } \\
\text { promedio }\end{array}$ & $\begin{array}{c}\text { Desviación } \\
\text { (DS) }\end{array}$ \\
\hline Proteína & $3,3 \%$ & 0,1 \\
Lactosa & $4,3 \%$ & 0,2 \\
Sólidos totales & $12,4 \%$ & 0,4 \\
Grasa & $4,1 \%$ & 0,3 \\
Sólidos no grasos & $8,3 \%$ & 0,2 \\
Peso específico & 1,0280 & 0,0005 \\
ATECAL (\%) & $0,17 \%$ & 0,03 \\
\hline
\end{tabular}

La composición promedio obtenida para la leche de cabra no difiere en peso específico y acidez de aquella comúnmente reportada por la literatura (Chacón 2005), donde el porcentaje de lactosa promedio de la leche de cabra es de 4,3\%. La misma se enmarca dentro de los márgenes establecidos por autores como Le Jaquen (1982), Pinto et al. (1984), Tacsan (1987) y Oliszewski et al. (2002), quienes reportan para la leche de cabra valores de sólidos totales promedio de 12,9 $\%$ (mínimo $11,5 \%$ ), grasa de 4,4 \% (mínimo 3,2 \%), lactosa de $4,9 \%$ (mínimo $4,0 \%$ ) y proteína de 3,5\% (mínimo 3,0 \%). El estudio de Chacón (2004) reporta, asimismo, un índice de ATECAL medio de la leche de cabra de $0,17 \%$ y un peso específico de 1,029.

Lo anterior permite afirmar que la leche empleada reúne la composición media aceptable para un procesamiento técnicamente viable y que, según la literatura, se encuentra dentro de los rangos normales de calidad (Brito 1989) y puede asociarse con adecuados rendimientos queseros (Oliszewski et al. 2002).

\section{Evaluación química del queso elaborado}

El Cuadro 2 presenta las características fisicoquímicas del queso evaluado en este trabajo.

Cuadro 2. Composición fisicoquímica promedio del queso de cabra derivado del "Crottin de Chavignol". San José, Costa Rica. 2008.

\begin{tabular}{lcc}
\hline \multicolumn{1}{c}{$\begin{array}{c}\text { Parámetro } \\
\text { evaluado }\end{array}$} & $\begin{array}{c}\text { Valor } \\
\text { promedio }\end{array}$ & $\begin{array}{c}\text { Desviación } \\
\text { (DS) }\end{array}$ \\
\hline $\begin{array}{l}\text { Rendimiento } \\
\text { Acidez (porcentaje de }\end{array}$ & $17 \%$ & 1 \\
ácido láctico) & $1,5 \%$ & 0,3 \\
Humedad & $56,0 \%$ & 0,9 \\
Materia seca & $43,9 \%$ & 0,5 \\
Proteína & $12 \%$ & 1 \\
Grasa (base húmeda) & $25,5 \%$ & 0,8 \\
Sal & $0,5 \%$ & 0,3 \\
\hline
\end{tabular}

Los resultados obtenidos son comparables con aquellos reportados por Tacsan (1987), para el queso en aquel entonces adaptado al gusto del consumidor costarricense y derivado del "Crottin de Chavignol". Dicha autora reportó valores de 55,3\% de humedad, $25,8 \%$ de grasa y $16,8 \%$ de proteína, así como un rendimiento medio de $17,7 \%$. De la comparación se puede evidenciar como el queso actual difiere de manera no muy acentuada con su antecesor. La relación inversa entre los parámetros de humedad y contenido de grasa en los quesos se encuentra ampliamente tipificada en la bibliografía existente (Corrales y Chacón 2005).

Parámetros como la humedad, la materia grasa y la acidez definen, por lo general, no solo la variedad a la que pertenece un queso, sino que en gran medida establecen las características técnicas y sensoriales del producto (Brito 1989). En función del contenido de humedad y de grasa es posible clasificar el queso sometido a estudio como "extra graso" (contenido mínimo del $40 \%$ de grasa en base seca) y como un queso "blando de corte" (entre el $54 \%$ y el $63 \%$ de 
humedad), similar al "Crottin de Chavignol" que se suele clasificar como un queso graso de pasta blanda (Tacsan 1987).

Los quesos que suelen catalogarse dentro de las categorías antes mencionadas son por lo general relativamente ácidos (Scholz 1995). La acidez obtenida por fermentación durante el tiempo prolongado de desuerado, de hecho puede explicar en parte el contenido de humedad intermedio de este queso en comparación con los quesos frescos (60\% - $80 \%$ humedad), dado que, como se expuso anteriormente en este documento, un $\mathrm{pH}$ ácido genera una mayor repulsión de agua en la fracción proteica (Walstra et al. 1999). A esto se suma la alta sinéresis que suele ser característica en los quesos caprinos (Calvo y Balcones 2000) y la porosidad de este tipo de cuajadas (Tacsan 1987). La variabilidad encontrada en el parámetro de acidez es un reflejo fiel de las condiciones artesanales con que es elaborado este producto, principalmente por dos motivos. Primero, la fermentación se realiza a temperatura ambiente, la cual, por su naturaleza, experimenta fluctuaciones que pueden incidir en la actividad metabólica de los cultivos. Segundo, pueden haber pequeñas variaciones en la cantidad del cultivo iniciador porque su dosificación se realiza de manera empírica.

La cantidad de sal obtenida permite clasificar el queso como uno bajo en sal (Brito 1985). No obstante, al ser el salado artesanal efectuado al gusto según los diferentes productores, este parámetro se considera en términos generales muy variable. Dado el bajo contenido de sal reportado en el queso evaluado, no es posible afirmar, para este caso particular, que ejerza una fuerza osmótica tal que sea un determinante primario en la eliminación del agua atrapada en la red proteica de la cuajada (Adda et al. 1982). Su papel en este caso puede estar más relegado a lo sensorial y a la preservación (Chacón 2003).

Los rendimientos obtenidos fueron estimados en función de los kilogramos de leche necesarios para obtener un kilogramo de queso (Brito 1985) y fueron consistentes con aquellos reportados por Oliszewski et al. (2002), autores que reportan un $16,50 \%$ de rendimiento para quesos similares de cabra. Este rendimiento es alto y apropiado desde un punto de vista comercial para desarrollos en la pequeña y mediana industria (Chacón 2003).

Por sus características fisicoquímicas, la literatura señala que es de esperar que el queso en estudio posea una vida útil en almacenamiento comparable con la de los quesos muy frescos, los cuales maduran con relativa rapidez (Scholz 1995), lo que favorece su fácil manufactura en medios artesanales.

\section{Evaluación del color}

El Cuadro 3 detalla las características de color obtenidas al evaluar el queso de cabra empleando un colorímetro. El producto evaluado tuvo una alta luminosidad $\left(\mathrm{L}^{*}\right)$, un valor muy bajo de $\mathrm{a}^{*}$ (casi imperceptible coloración verde) y un valor de $b^{*}$ de 10 ; lo que indica una leve coloración amarilla. La razón de lo anterior encuentra fundamento en el bajo contenido de carotenoides de la leche de cabra, razón que impide que la misma presente un color amarillento similar al de la leche vacuna, caracterizándose los quesos frescos caprinos por su color más blanco en relación con los vacunos (Chacón 2005). El ángulo de tonalidad se encuentra en los $100,6^{\circ}$, que es la zona del amarillo al verde, color que casi no es perceptible en el queso debido a la alta luminosidad y a la poca saturación $\left(\mathrm{C}^{*}\right)$ del color. Según Álvarez et al. (2007), esto se debe a que los quesos con alto contenido de humedad tienden a ser más luminosos y menos saturados. Además, todas las variables de color determinadas presentan una variabilidad muy baja, lo que indica que, a pesar de que el queso tipo Crottin evaluado se fabrica de forma artesanal, su color se mantiene considerablemente constante entre los diferentes lotes de producción.

Cuadro 3. Características promedio de color del queso de cabra derivado del "Crottin de Chavignol" para el espacio polar CIE. San José, Costa Rica. 2008.

\begin{tabular}{ccc}
\hline $\begin{array}{c}\text { Parámetro } \\
\text { evaluado }\end{array}$ & Valor promedio & $\begin{array}{c}\text { Desviación } \\
\text { (DS) }\end{array}$ \\
\hline $\mathrm{L}^{*}$ & 92,4 & 0,3 \\
$\mathrm{a}^{*}$ & $-1,9$ & 0,3 \\
$\mathrm{~b}^{*}$ & 10 & 1 \\
$\mathrm{C}^{*}$ & 10 & 1 \\
$\mathrm{~h}^{*}$ & 100 & 1 \\
\hline
\end{tabular}

$\mathrm{L}^{*}$ : variación en la luminosidad (variando desde $0 \%$ para negro y $100 \%$ para blanco), a*: variación entre verde (-a) y rojo $(+a)$, $\mathrm{b}^{*}$ : variación entre azul (-b) y amarillo $(+b), C^{*}$ : saturación del color, $\mathrm{h}^{*}$ : ángulo de tonalidad. 
Adicionalmente, las características de color obtenidas son muy similares a las reportadas por Saldo et al. (2002) y Saldo et al. (2001) en un queso duro de cabra, lo que demuestra la tendencia de varios tipos de queso de cabra a presentar coloraciones muy blancas.

\section{Evaluación de la textura}

En la Figura 5 se presenta el perfil de textura del queso de cabra, mientras que el Cuadro 4 detalla las características de textura obtenidas. La elasticidad y la cohesividad presentaron poca variabilidad, en tanto que la dureza, la masticabilidad y la adhesividad resultaron altamente variables en el queso de cabra analizado en este trabajo. Tal y como señala Castro (2007), los parámetros texturales pueden tener mucha variabilidad, considerándose precisos los valores de coeficiente de variabilidad menores a $10 \%$. El autor anteriormente mencionado también indica que el análisis reológico del fenómeno de deformaciones, como es el caso de la prueba de compresión, es bastante complejo, debido a que las estructuras no son uniformes y tienen grandes variaciones naturales.

El perfil de textura de un alimento está definido por varios parámetros (Pinho et al. 2004). En términos generales, la dureza es la fuerza necesaria para provocar una cierta deformación en el queso, constituyendo el pico máximo de fuerza durante la primera compresión; por su lado, la elasticidad corresponde a cómo se comporta el producto al tratar de regresar a su forma original con el tiempo, aspecto que generalmente se asocia con el contenido de grasa del queso.

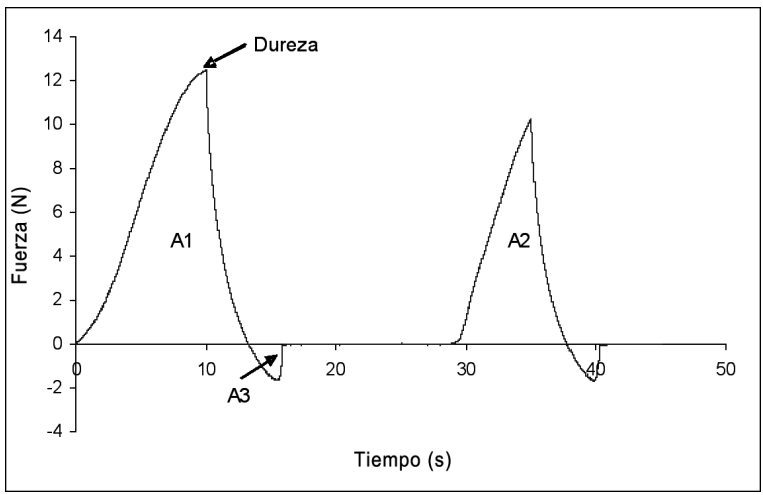

Figura 5. Perfil de textura característico del queso de cabra derivado del "Crottin de Chavignol". San José, Costa Rica. 2008.
Cuadro 4. Características de perfil de textura del queso de cabra derivado del "Crottin de Chavignol". San José, Costa Rica. 2008.

\begin{tabular}{ccc}
\hline $\begin{array}{c}\text { Parámetro } \\
\text { evaluado }\end{array}$ & Valor promedio & $\begin{array}{c}\text { Desviación } \\
\text { Estándar (DS) }\end{array}$ \\
\hline Dureza $(\mathrm{N})$ & 10 & 3 \\
Elasticidad & 0,72 & 0,08 \\
Cohesividad & 0,52 & 0,06 \\
Masticabilidad & 0,03 & 0,01 \\
$(\mathrm{Nm})$ & & \\
Adhesividad & $-1,2$ & 0,7 \\
$(\mathrm{Nm}) * 10^{-3}$ & & \\
\hline
\end{tabular}

La cohesividad del queso se determina con base en el comportamiento durante la segunda compresión en comparación a cómo se comportó durante la primera (relación A2/A1) y representa el punto límite hasta el cual puede deformarse el material antes de romperse (Osorio et al. 2004). La masticabilidad denota la energía necesaria para masticar el alimento y es definida en términos del producto de la multiplicación de la dureza, la elasticidad y la cohesividad. Finalmente, la adhesividad (A3) es la fuerza necesaria para superar la de atracción entre la superficie del alimento y aquellos materiales con los que entra en contacto, en este caso la sonda del texturómetro.

El queso de cabra preparado presentó una dureza promedio de $10 \mathrm{~N}$. La dureza de éste está relacionada ampliamente con su composición química. La humedad es un factor determinante en la textura final, donde bajos contenidos se asocian con quesos duros y poco elásticos (Adda et al. 1982). Como se explicó con anterioridad, la grasa, cuando está presente en alto porcentaje, brinda mayor elasticidad y menor firmeza, obteniéndose quesos más duros y rígidos al bajar este porcentaje (Küçüköner y Haque 2006, Theophilou y Wilbey 2007). Por lo tanto, por su contenido de humedad y grasa el queso de cabra obtenido se clasifica como un queso blando. Otros factores como el método de preparación y el grado de madurez también influyen en las características texturales de los quesos. Phadungath (2005) menciona que se han hallado cinco condiciones de proceso que, principalmente, afectan la textura y propiedades sensoriales del queso crema: el contenido de grasa en la leche estandarizada, la presión de homogenización, el nivel de inóculo, la 
temperatura de incubación y el pH en el momento de romperse el gel lácteo. Otros factores que influencian las propiedades físicas y el aroma del queso son: la calidad de la leche y su composición, la extensión de la acidificación por parte de las bacterias iniciadoras, la concentración de sales de calcio (proporciones de las formas solubles e insolubles), la extensión de la proteólisis y otras reacciones ocurridas durante la maduración (Lucey 2003).

Sin embargo, en la determinación instrumental de la textura, el método utilizado (tipo y tamaño de la sonda, velocidad de la prueba, tipo de prueba -compresión o penetración-, tamaño y forma de la muestra) también tienen gran influencia en los resultados obtenidos, los que solo son comparables cuando han sido realizados exactamente en las mismas condiciones de medición. Al respecto, Bourne (2002) recomienda estandarizar todas las condiciones de la prueba con el fin de obtener la mejor resolución entre las diferentes muestras evaluadas. Debido a que la cohesividad es adimensional y la elasticidad puede transformarse en un valor relativo, son los únicos dos parámetros de textura medidos cuyos valores son comparables a los obtenidos de otros estudios con condiciones experimentales diferentes. Bourne (1982) afirma que, aunque la elasticidad solo se puede comparar entre productos con idéntica forma y tamaño, al expresar la elasticidad como una razón de la altura original de la muestra, pueden hacerse comparaciones entre una más amplia variedad de muestras y productos.

Bajo las condiciones en que se realizó la prueba, el queso de cabra resultó bastante elástico, pues recobró el $71,9 \%$ de su altura original luego de la primera penetración. Esto implica que, a pesar de tratarse de un queso de pasta blanda, con alto contenido de humedad, y estructura aparentemente frágil, es más elástico que varios tipos de queso reportados por diversos autores, como el Edam, el queso fresco y el Panela (Cuadro 5). La elasticidad del queso obtenido se puede explicar porque es un queso fresco, que tiene una menor cantidad de cadenas proteicas de bajo peso molecular, las cuales se producen con el tiempo debido a diversos factores como la acción de los microorganismos y que, por su pequeño tamaño, no pueden interactuar de igual forma con las cadenas de mayor tamaño, lo cual genera una disminución de la elasticidad con el tiempo (Serafin 1999).

El queso de cabra posee una cohesividad intermedia pues, en promedio, el área de la segunda curva
(A2) es apenas un poco mayor a la mitad del área de la primera curva (A1). En comparación, este queso es menos cohesivo que los Panela, fresco y Mozzarella bajo en grasa, pero mucho más cohesivo que el Edam y el Cheddar (Cuadro 5). Según Álvarez et al. (2007), la cohesividad y el contenido de humedad presentan una correlación positiva, por lo cual, a mayor contenido acuoso, mayor es la fuerza ejercida por los enlaces internos del queso. Como generalmente la humedad de los quesos disminuye con la maduración (Alais 1984, Revilla 1985), es de esperar que la cohesividad de los quesos frescos sea mayor que la de los quesos madurados o que tienen algún tiempo de estar almacenados, tal y como lo observaron Van Hekken et al. (2002) en un queso suave de cabra. Adicionalmente, conforme aumenta la elasticidad del producto, aumenta también la

Cuadro 5. Elasticidad y cohesividad de diversos tipos de quesos preparados con leche bovina. San José, Costa Rica. 2008

\begin{tabular}{|c|c|c|c|}
\hline $\begin{array}{c}\text { Tipo de queso/ } \\
\text { Tiempo }^{\text {a }}\end{array}$ & $\begin{array}{l}\text { Elastici- } \\
\operatorname{dad}^{b}\end{array}$ & $\begin{array}{c}\text { Cohesivi- } \\
\text { dad }\end{array}$ & Referencia \\
\hline Fresco c/una semana & 0,643 & 0,78 & $\begin{array}{c}\text { Farkye } e t \\
\text { al. } 1995\end{array}$ \\
\hline Edam/30 días & 0,296 & 0,2370 & $\begin{array}{l}\text { Osorio et } \\
\text { al. } 2005\end{array}$ \\
\hline $\begin{array}{l}\text { Panela (leche entera)/ } \\
\text { tres días }\end{array}$ & 0,509 & 0,8 & $\begin{array}{l}\text { Lobato-Ca- } \\
\text { lleros et al. } \\
\quad 2006\end{array}$ \\
\hline $\begin{array}{l}\text { Panela (bajo en } \\
\text { grasa)/tres días }\end{array}$ & 0,691 & 0,8 & $\begin{array}{l}\text { Lobato-Ca- } \\
\text { lleros et al. } \\
\quad 2006\end{array}$ \\
\hline Mozzarella/semana 0 & 0,89 & 0,61 & $\begin{array}{c}\text { Imm et al. } \\
2003\end{array}$ \\
\hline $\begin{array}{l}\text { Mozzarella } \\
(25 \% \text { grasa)/tres días }\end{array}$ & 0,522 & 0,47 & $\begin{array}{l}\text { Rudan et } \\
\text { al. } 1999\end{array}$ \\
\hline $\begin{array}{l}\text { Mozzarella } \\
(5 \% \text { grasa)/tres días }\end{array}$ & 0,757 & 0,71 & $\begin{array}{l}\text { Rudan et } \\
\text { al. } 1999\end{array}$ \\
\hline Cheddar/un día & 0,75 & 0,42 & $\begin{array}{l}\text { Serrano et } \\
\text { al. } 2004\end{array}$ \\
\hline \multicolumn{4}{|c|}{$\begin{array}{l}\text { a Tiempo transcurrido entre la elaboración del queso y el análisis } \\
\text { b Valor calculado al dividir la altura recuperada por la muestra luego } \\
\text { de la primera compresión del TPA por la distancia de compresión. } \\
\text { Para los quesos estudiados por Imm et al. (2003) y Rudan et al. } \\
\text { (1999), la razón de alturas fue reportada directamente. } \\
\text { c Producido por acidificación con ácido láctico. }\end{array}$} \\
\hline
\end{tabular}

AGRONOMÍA MESOAMERICANA 20(2):297-309. 2009 
resistencia a la deformación del alimento por la flexibilidad de los enlaces internos (Muller 1977), por lo que es de esperar que también aumente la cohesividad.

Para la masticabilidad, el queso de cabra en estudio presentó valores acordes con los reportados para otros tipos de queso. Por otro lado, éstos no son productos particularmente adhesivos, por lo que esta característica no se reporta en muchos estudios. Sin embargo, dentro de lo reportado y, como indican Álvarez et al. (2007), debido a su contenido de grasa este queso puede resultar más adhesivo que otros tipos. La adhesividad también aumenta con el tiempo de maduración (Osorio et al. 2004) pero disminuye con el contenido de humedad (Álvarez et al. 2007).

\section{Prueba sensorial afectiva}

La evaluación por parte de jueces no entrenados del queso de cabra tipo "Crottin" y uno de crema comercial dio como resultado dos grupos de personas (Figura 6). El $55 \%$ (24 jueces) quedó incluido en el conglomerado 1 , el que se caracteriza por tener un agrado similar y alto por los dos tipos de queso. El otro $45 \%$ (20 jueces) se ubicó en el grupo 2; a las personas de este grupo les agradó mucho el queso crema pero manifestaron disgusto por el de cabra. Aunque es necesario realizar más estudios con consumidores, estos resultados permiten tener evidencia de que existe una porción apreciable del mercado costarricense que percibe el queso tipo Crottin tan agradable como el crema elaborado con leche vacuna, lo cual representa

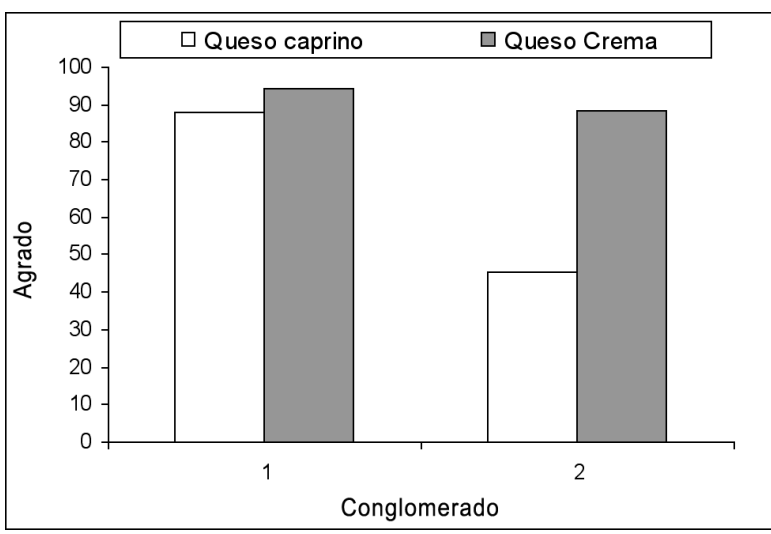

Figura 6. Resultados de la prueba de aceptación para el queso de cabra y el queso crema. San José, Costa Rica. 2008 buenas opciones para la comercialización de ese tipo de queso de cabra. En el estudio de Corrales y Chacón (2005) también se evidenció que los consumidores costarricenses presentan una buena aceptación por el queso fresco de cabra.

Los resultados anteriores son de especial interés, sobretodo si se tiene la intención de promover el consumo de derivados lácteos caprinos en un mercado como el costarricense, donde un estudio básico del consumidor efectuado por Chacón et al. (2008) reveló que un 93,7 \% de las personas evaluadas no consumen leche caprina y solamente un 11,24\% de dicha población consumen derivados de ésta, especialmente queso y yogurt. El corroborar que el queso caprino evaluado posee un adecuado desempeño sensorial al compararlo con un producto similar más tradicional y disponible en el mercado, como el queso crema elaborado con leche vacuna, proyecta al mismo con un producto con buenas posibilidades de trascender la zona geográfica donde es elaborado de manera artesanal. Esta última razón también justificaría los esfuerzos de caracterización y estandarización efectuados en los últimos años para con este producto.

\section{LITERATURA CITADA}

Adda, J; Gripon, JC; Vassal, L. 1982. The chemistry of flavour and texture generation in cheese. Food Chemistry 9(1):115-129.

Alais, C. 1984. La ciencia de la leche. CECSA, México, D.F. 477 p.

Álvarez, S; Rodríguez, V; Ruiz, ME; Fresno, M. 2007. Correlaciones de textura y color instrumental con la composición química de quesos de cabra canarios. Archivos de Zootecnia 56 (Sup. 1):663-666.

AOAC. (Association of Official Analytical Chemistry). 2000. Official methods of analysis. Association of Official Analytical Chemistry, Washington D.C. Capítulo 33:844.

Armero, E; Collar, C. 1997. Texture properties of formulated wheat doughs. Relationships with dough and bread technological quality. Lebensm Unters Forsch. 204:136-145.

Bara-Herczegh, O; Horvath-Almassy, K; Fenyvessy, J; Orsi, F. 2002. Suitability of texture parameters for characterization of Hajdu cheese ripening. Acta Alimentaria 31(2):149-159.

Bourne, MC. 1982. Food Texture and Viscosity. Academic Press, New York. 330 p. 
Bourne, MC. 2002. Food Texture and Viscosity: Concept and Measurement. Academic Press, New York. 416 p.

Brito, C. 1985. Aspectos tecnológicos y caracterización del queso chaco de campo. Alimentos 10(3):41-46.

Brito, C. 1989. Miti-Miti: nueva variedad de queso de cabra. Alimentos 14(4):7-15.

Calvo, MM; Balcones, E. 2000. Some factors influencing the syneresis of bovine, ovine and caprine milks. Journal of Dairy Science 83 (8):1733-1739.

Carpenter, RP; Lyon, DH; Hasdell, TA. 2000. Guidelines for sensory analysis in food product development and quality control. Aspen Publishers, New York. 212 p.

Castro, E. 2007. Parámetros mecánicos y textura de los alimentos. Universidad de Chile, Santiago, Chile. 144 p.

Chacón, A. 2003. La elaboración del queso fresco y otros derivados lácteos: guía básica artesanal y de la pequeña industria. Ed. Universidad de Costa Rica. San Pedro de Montes de Oca, San José. 66 p.

Chacón, A. 2004. Acidez y peso específico de la leche de cabra de un grupo de capricultores de la Meseta Central Costarricense. Agronomía Mesoamericana 15(2):179-183.

Chacón, A. 2005. Aspectos nutricionales de la leche de cabra y sus variaciones en el proceso agroindustrial. Revista Agronomía Mesoamericana 16(2):239-252.

Chacón, A; Araya, YM; Gamboa, ME. 2008. Percepciones y hábitos de consumo de la leche de cabra y sus derivados en los costarricenses. Agronomía Mesoamericana 19(2):241-250

Corrales, J; Chacón, A. 2005. Estudio de opinión de consumidores sobre el queso fresco de cabra (Capra hircus) en Costa Rica. Revista de Agricultura Tropical 35:39-49.

Devoyod, JJ. 1991. Crottin de Chavignol: a multi-facetted cheese. Ethnozootechnie 47(1):31-38.

Díaz, C. 2004. Caracterización de la agroindustria láctea en Turrialba. Costa Rica. Revista de Agricultura Tropical 34:27-39.

Farkye, NY; Prasad, BB; Rossi, R; Noyes, OR. 1995. Sensory and textural properties of queso blanco-type cheese influenced by acid type. Journal of Dairy Science 78(8):1649-1656.

HUNTERLAB. 2008. CIE L* C* h color scale. Applications Note 8(11): 1-4.

Imm, JY; Oh, EJ; Han, KS; Oh, S; Park, YW; Kim, SH. 2003. Functionality and physico-chemical characteristics of bovine and caprine mozzarella cheeses during refrigerated storage. Journal of Dairy Science 86(9):2790-2798.
Kirk, RS; Sawyer, R; Egan, E. 1999. Composición y análisis de los alimentos de Pearson. 2 ed. Continental, México D.F. 777 p.

Kozhev, A; Spasova, M; Kozhev, S. 1998. Goat cheese an alternative product on the market or a gastronomic exoticism? Kkranitelnovkusova Promishlenost 47(9):14-15.

Küçüköner, E; Haque ZU. 2006. Physicochemical properties of low-fat and full-fat Cheddar cheeses. International Journal of Dairy Technology 59(3):166-170.

Le Jaquen, JC. 1982. La fabrication du fromage de chèvre fermier. 3 ed. Societé de Presse et d'Edition Ovine et Caprine. Paris, Francia. 209 p.

Lobato-Calleros, C; Ramos-Solís, L; Santos-moreno, A; Rodríguez-Huezo, ME. 2006. Microstructure and texture of panela type cheese-like products: use of low methoxyl pectin and canola oil as milk-fat substitutes. Revista Mexicana de Ingeniería Química 5:71-79.

Lucey, JA; Johnson, ME; Horne, DS. 2003. Perspectives on the basis of the rheology and texture properties of cheese. Journal of Dairy Science 86(9):2725-2743.

Morón, C; Alonso, L; Crovetto, M. 2005. Cambios en la estructura del consumo de alimentos y nutrientes de América Latina 1979-1981 a 1999-2001. Organización de las Naciones Unidas para la Agricultura y la Alimentación (FAO), Oficina Regional de la FAO para América Latina y el Caribe. Santiago de Chile. 63 p.

Muller, HG. 1977. Introducción a la reología de los alimentos. Acribia. Zaragoza, España. 174 p.

Oliszewski, R; Rabasa, A; Fernández, JL; Poli, M; Núñez De Kairúz, M. 2002. Composición química y rendimiento quesero de la leche de cabra Criolla Serrana del noroeste argentino. Zootecnia Tropical 20(2):179-189.

Osorio, JP; Ciro, HJ; Mejía, LG. 2004. Caracterización textural y fisicoquímica del queso EDAM. Revista Facultad Nacional de Agronomía, Medellín 57(1): 2275-2286.

Osorio, JF; Ciro, HJ; Mejía, LG. 2005. Caracterización reológica y textural del queso Edam. Dyna 72(147):33-45.

Phadungath, C. 2005. Cream cheese products: A review. Songklanakarin Journal of Science and Technology 27(1):191-199.

Pinho, O; Mendes, E; Alves, MM; Ferreira, IMPLVO. 2004. Chemical, physical, and sensorial characteristics of "Terrincho" ewe cheese: Changes during ripening and intravarietal comparison. Journal of Dairy Science 87(2):249-257.

Pinto, M; Villena, J; Jofre, H. 1984. Contribución al estudio de la composición de la leche de cabra Anglo-Nubian. Agro-Sur 12(2):163-173.

AGRONOMÍA MESOAMERICANA 20(2):297-309. 2009 
Revilla, A. 1985. Tecnología de la leche. IICA, San José. $598 \mathrm{p}$.

Rojas, WN; Chacón, A; Pineda, ML. 2007. Características del yogurt batido de fresa derivadas de diferentes proporciones de leche de vaca y cabra. Agronomía Mesoamericana 18(2):221-237.

Rudan, MA; Barbano, DM; Yun, JJ; Kindstedt, PS. 1999. Effect of fat reduction on chemical composition, proteolysis, functionality, and yield of Mozzarella cheese. Journal of Dairy Science 82(4):661-672.

Saldo, J; Sendra, E; Guamis, B. 2001. Hard cheese structure after a high hydrostatic pressure treatment at $50 \mathrm{MPa}$ for 72 $\mathrm{h}$ applied to cheese after brining. Lait 81(5):625-635.

Saldo, J; Sendra, E; Guamis, B. 2002. Colour changes during ripening of high pressure treated hard caprine cheese. High Pressure Research 22(3-4):659-663.

Scholz, W. 1995. Elaboración de quesos de oveja y de cabra. Acribia. Zaragoza, España. 145 p.

Serafin, AZ. 1999. Use of a vibration technique to monitor the textural properties of cheese. Tesis M.Sc. Faculty of Graduate Studies, University of Guelph. Ontario. 98 p.

Serrano, J; Velazquez, G; Lopetcharat, K; Ramírez, JA; Torres, JA. 2004. Effect of moderate pressure treatments on microstructure, texture, and sensory properties of stirred-curd cheddar shreds. Journal of Dairy Science 87(10):3172-3182.

Steffe, JF. 1996. Rheological methods in food process engineering. 2 ed. Freeman Press, East Lansing, Michigan. $418 \mathrm{p}$.
Tacsan, I. 1987. Fabricación de un queso de leche de cabra tipo crottin adaptado al gusto costarricense. Tesis Lic. en Tecnología de Alimentos. Universidad de Costa Rica, Escuela de Tecnología de Alimentos. San José. $83 \mathrm{p}$.

Theophilou, P; Wilbey, RA. 2007. Effects of fat on the properties of halloumi cheese. International Journal of Dairy Technology 60(1):1-4.

Van Hekken, DL; Tunick, MH; Park, YW. 2002. Effect of frozen storage on the proteolysis and rheology of soft goat milk cheese. Journal of Animal Science 80/Journal of Dairy Science 85(Suppl. 1):254(Abstr.).

Vargas, P; Pineda, ML; Chacón, A. 2007. Lácteos bovinos y percepción de la leche caprina entre estudiantes de la Universidad de Costa Rica. Agronomía Mesoamericana 18(1):27-36.

Vega, O. 2002. Desempeño de la ganadería de leche y de la industria de la transformación de productos lácteos en Costa Rica 1999-2001. SEPSA, Área de Estudios Económicos e Información. San José. 32 p.

Villanueva, NDM; Petenate, AJ; Da Silva, MAAP. 2005. Performance of the hybrid hedonic scale as compared to the traditional hedonic, self-adjusting and ranking scales. Food Quality and Preference 16(8):691-703.

Walstra, P; Geurts, TJ; Noomen, A; Jellema, A; Van Boekel, MAJS. 1999. Dairy Technology, principles of milk, properties and processes. Marcel Dekker, New York. $727 \mathrm{p}$. 
